S Aliberti

JA Myers

P Peyrani

F Blasi

R Menendez

P Rossi

R Casentini

G Lopardo

L de Vedia

JA Ramirez

\section{O papel da neutropenia no prognóstico do doente oncológico com pneumonia adquirida na comunidade*}

\section{The role of neutropenia on outcomes of cancer patients with community-acquired pneumonia*}

\section{Resumo}

A doença infecciosa contribui para uma elevada morbilidade e mortalidade no doente oncológico, representando a pneumonia adquirida na comunidade a mais frequente.

O desenvolvimento de PAC no doente neoplásico parece advir da modificaçáo de mecanismos de defesa imunitária resultante, quer da patologia maligna, quer do tratamento oncológico. O risco de infecção relacionada com o tipo de neoplasia pode associar-se ao défice de imunidade humoral, celular ou do número de neutrófilos. As doenças hematológicas malignas podem predispor o doente às infecçóes devido à substituição da medula por células neoplásicas. Consequentemente, estes doentes têm neutropenia funcional, apesar de apresentarem, muitas vezes, um número normal ou aumentado de neutrófilos. Por outro lado, estes doentes podem ter neutropenia como efeito secundário da quimioterapia e/ou radioterapia (neutropenia absoluta).

A gravidade da neutropenia foi considerada como principal factor de risco isolado no doente neoplási- co, com particular relevância se o número de neutrófilos $\leq 500 \mathrm{cel} / \mathrm{mm}^{3}$.

A mortalidade global atribuída à neutropenia febril no doente neoplásico é de 30-50\%. Nas últimas décadas, o tratamento das infecçóes na população oncológica foi direccionado, primariamente, para o manuseamento da neutropenia febril, devido ao facto de o local da infecção não ser determinado em 50-80\% dos casos. As guidelines da American Thoracic Society de 2001 utilizavam a neutropenia para identificar os quadros mais graves de PAC nos doentes oncológicos. Os doentes com patologia hematológica e neutropenia funcional ou indivíduos com qualquer tipo de neoplasia e neutropenia absoluta foram excluídos das referidas guidelines. A decisão de incluir doentes com tumores sólidos não neutropénicos foi baseada, apenas, na opinião de especialistas. Assim, os clínicos podiam sentir-se confiantes e tratar a PAC no doente oncológico não neutropénico como na população geral. No entanto, o papel da neutropenia no prognóstico de doentes neoplásicos hospitalizados com PAC não tinha, ainda, sido investigado

* Eur Respir J 2009; 33:142-147 
O objectivo do presente estudo foi comparar o prognóstico de doentes neoplásicos e doentes imunocompetentes com PAC, bem como o prognóstico de indivíduos com patologia maligna e neutropenia absoluta ou funcional e sem neutropenia.

Foi efectuada uma análise retrospectiva envolvendo 43 hospitais em 12 países no período compreendido entre Junho de 2001 e Janeiro de 2006. Foram incluídos os indivíduos com idade $\geq 18$ anos com critérios de PAC, tendo sido eliminados os portadores de infecção VIH. A PAC foi definida pela presença de infiltrado pulmonar "de novo" na radiografia do tórax na altura do internamento, associado a, pelo menos, um dos seguintes parâmetros: tosse ou aumento da intensidade desta; $\operatorname{tax}<35,6^{\circ} \mathrm{C}$ ou $>37,8^{\circ} \mathrm{C}$; número de leucócitos no sangue periférico (leucocitose, desvio esquerdo ou leucopenia).

Neoplasia foi definida como qualquer tipo de malignidade diagnosticada nos 12 meses anteriores ou neoplasia activa. Considerou-se que esta estaria presente em doentes sob quimioterapia e/ou radioterapia nos 12 meses precedentes ou com sinais ou sintomas de neoplasia ao longo do último ano. Doentes com neoplasia de células escamosas ou basais da pele foram consideradas imunocompetentes.

A neutropenia foi classificada como funcional ou absoluta. A primeira estava presente em doentes com doença hematológica maligna. A neutropenia absoluta correspondeu a um número de neutrófilos $<500 \mathrm{cel} /$ $/ \mathrm{mm}^{3}$ na admissão hospitalar.

Os doentes com PAC foram divididos em dois grupos: doentes não oncológicos - Grupo I, e doentes com neoplasia - Grupo II. Este último foi, por seu turno, subdividido em dois subgrupos, de acordo com o tipo de neoplasia e o número de neutrófilos - doentes com tumor sólido sem neutropenia (Grupo IIa) e doentes com neutropenia (Grupo IIb).

Foram avaliados a mortalidade, a duração do internamento e o tempo de estabilização clínica.

Foram estudados 3106 indivíduos com PAC dos quais 135 eram doentes neoplásicos sem neutropenia e 75 tinham neoplasia e neutropenia.

A taxa de mortalidade foi significativamente superior nos doentes oncológicos em comparação com os doentes não neoplásicos (14\% versus $8 \%)$. Nestes últimos, a morte foi atribuída à PAC em $30 \%$ dos casos. A presença de neoplasia na população estudada teve impacto significativo na mortalidade quando ajustado para as covariáveis.

Os doentes com neoplasia também tiveram um tempo de estabilização clínica significativamente superior ( $5,1 \pm 2,6$ dias versus $4,6 \pm 2,5$ dias) e um tempo de internamento mais prolongado $(9,3 \pm 4,8$ dias versus $8,4 \pm 4,7$ dias).

Nos doentes neoplásicos com ou sem neutropenia, o tempo de estabilização clínica foi semelhante nos dois subgrupos $(5,7 \pm 2,7$ versus $4,9 \pm 2,7$ dias, respectivamente), bem como o tempo médio de internamento (9,2 $2 \pm 7,7$ versus $9,9 \pm 9,6$ dias, respectivamente).

Não se verificou uma diferença estatisticamente significativa na taxa de mortalidade dos doentes oncológicos com ou sem neutropenia (18\% versus $15 \%$ ), mas esta diferença já foi significativa quando comparados indivíduos não neoplásicos com os doentes neoplásicos sem neutropenia ( $8 \%$ versus $15 \%)$ e com neutropenia ( $8 \%$ versus $18 \%)$. 


\section{Comentário}

Este estudo indica que, em doentes com PAC, a presença de malignidade aumenta significativamente a mortalidade e piora o prognóstico. Verificou-se, no entanto, que nos doentes oncológicos, este último era similar nos indivíduos com ou sem neutropenia. A presença desta na admissão hospitalar não constitui, de forma independente, um factor de risco de mortalidade nos doentes neoplásicos com PAC. Isto sugere, também, que os clínicos devem tratar de forma contundente os portadores de neoplasia com PAC, independentemente do tipo de tumor ou do número de neutrófilos à entrada no hospital. A ausência de neutropenia na avaliação inicial nestes indivíduos não deve ser considerada um indicador do melhor prognóstico clínico.

Apesar de a neutropenia ser um factor de risco para infecçóes, outros parâmetros parecem constituir nos doentes oncológicos com PAC um factor de risco equivalente para um agravamento do prognóstico.

Sabe-se que indivíduos com tumores sólidos podem ter uma contagem de neutrófilos normal e, no entanto, os fármacos citostáticos usados terem afectado a função fagocítica e quimiotáctica dos mesmos. Por outro lado, a capacidade fagocítica dos neutrófilos, eosinófilos e células mononucleadas pode estar intrinsecamente alterada, mesmo antes do início de quimioterapia. Os mecanismos de defesa imunitária humorais e celulares mediados pelos linfócitos $\mathrm{B}$ e $\mathrm{T}$, respectivamente, podem estar profundamente afectados nos doentes com tumores sólidos. Assim, múltiplos aspectos do sistema de defesa do hospedeiro podem estar, simultaneamente, modificados nos indivíduos com patologia maligna e PAC internados, condicionando, assim, o prognóstico.
Usando um modelo regressivo de logística, a neutropenia não está significativamente associada à mortalidade no doente oncológico quando ajustada para covariáveis significativas. $\mathrm{O}$ mesmo não sucede com a classificação CRB-65 (confusão, frequência respiratória $\geq 30 \mathrm{cpm}$; PA sist. $<90 \mathrm{mmHg}$ ou PA diast. $\leq 60 \mathrm{mmHg}$ e idade $\geq 65$ anos) que está associa$\mathrm{da}$, independentemente, à mortalidade.

Outros estudos efectuados em doentes internados em unidades de cuidados intensivos demonstraram que a neutropenia não está significativamente associada à mortalidade em doentes oncológicos com infecçôes de diferentes origens. Nas últimas décadas, o manuseamento da infecção em doentes oncológicos estava centrada na presença de febre e neutropenia. No entanto, as ultimas guidelines publicadas pela National Comprehensive Cancer Network recomendam que os doentes imunocomprometidos náo neutropénicos recebam o mesmo tratamento dos indivíduos com neutropenia.

Uma importante limitação do presente trabalho na avaliaçáo do efeito da neutropenia no prognóstico é a ausência de dados sobre o tempo de duração da neutropenia antes da admissão hospitalar, o desenvolvimento e a recuperação da neutropenia durante o internamento.

Tratando-se de um estudo retrospectivo, não foi possível obter informação suplementar sobre a patologia maligna, a quimioterapia efectuada ou o transplante de medula óssea. Assim, os clínicos não podem assegurar um melhor prognóstico quando a neutropenia não está presente na avaliação inicial do doente neoplásico com PAC. Deste modo, os clínicos devem tratar agressivamente todos os indivíduos com neoplasia e PAC, independentemente do tipo de tumor e da contagem de neutrófilos. 


\section{Mensagem}

- Nos doentes com pneumonia adquirida na comunidade (PAC), a presença de patologia maligna aumenta significativamente a mortalidade e piora o prognóstico;

- Nos doentes oncológicos com PAC, o prognóstico é similar nos indivíduos com ou sem neutropenia;

- A presença de neutropenia na admissão hospitalar não é um factor de risco independente na mortalidade de doentes neoplásicos com PAC.

\section{Bibliografia}

1. Mandell LA, Wunderink RG, Anzueto A, et al. Consensus guidelines on the management of communityacquired pneumonia in adults. Clin Infect Dis 2007; 44(Supl 2):527-572.

2. National Comprehensive Cancer Network. Prevention and treatment of cancer-related infections, 2007 (www.nccn.org/about/news/newsimpo.asp?news ID=107).

3. Pizzo PA. Fever in immunocompromised patients. N Engl J Med 1999; 341: 893-900.

4. Rañó A, Agustí C, Benito N, et al. Prognostic factors of non-HIV immunocompromised patients with pulmonary infiltrates. Chest 2002; 122: 253-261.

Fátima Caeiro 2009-03-10 